\title{
On Importance of Rows for Decision Tables
}

\author{
Hassan AbouEisha, Mohammad Azad, and Mikhail Moshkov \\ Mathematical and Computer Sciences \& Engineering Division \\ King Abdullah University of Science and Technology \\ Thuwal 23955-6900, Saudi Arabia \\ \{hassan.aboueisha, mohammad.azad, mikhail.moshkov\}@kaust.edu.sa
}

\begin{abstract}
In this paper, we propose a method for the evaluation of importance of rows for decision tables. It is based on indirect information about changes in the set of reducts after removing the considered row from the table. We also discuss results of computer experiments with decision tables from UCI Machine Learning Repository.
\end{abstract}

Keywords: decision table, canonical form, test, reduct, characteristic function

\section{Introduction}

In this paper, we discuss a way to evaluate the importance of rows for decision tables (importance of objects for datasets) which is based on the study of changes in the set of reducts $[3,4]$ if we remove the considered row from the decision table. Unfortunately, the problem of constructing the set of reducts for a given decision table is too complicated: the number of reducts can grow exponentially depending on the size of the decision table. However, we can obtain useful indirect information about the set of reducts in polynomial time depending on the size of the input decision table.

We associate a given decision table $T$ with $n$ conditional attributes a characteristic function $f_{T}$ with $n$ variables that describes the set of tests (superreducts) for $T$ [2]. This is a monotone Boolean function for which the set of lower units correspond to the set of reducts for $T$. This function can be described not only by the set of lower units but also by the set of upper zeros $U_{T}$ which can be constructed for $T$ in polynomial time depending on the size of the input decision table. Note that upper zeros correspond to maximal subsets of attributes which are not tests. For a given row $r$ of the table $T$, we construct the table $T(r)$ obtained from $T$ by the removal of the row $r$. We find the cardinality of the symmetric difference of the sets $U_{T}$ and $U_{T(r)},\left|\left(U_{T} \cup U_{T(r)}\right) \backslash\left(U_{T} \cap U_{T(r)}\right)\right|$, which is considered as the importance of the row $r$ for the table $T$.

In [2], a way to find the set of upper zeros $U_{T}$ for the characteristic function $f_{T}$ was proposed for decision tables with binary attributes. It was used in creation of classifiers. In this paper, we extend it to arbitrary decision tables with categorical attributes, and use it in the evaluation of the importance of rows. 
The created technique can be useful for analysis of decision tables with categorical attributes. It will allow us to point to rows (objects) that have the greatest influence on the formation of the set of reducts. We apply this technique to a number of decision tables from the UCI Machine Learning Repository [1] and find the importance of rows for these tables.

The paper consists of fours sections. In Sect. 2, we consider the main notions, and discuss a way to find the set of upper zeros of the characteristic function and to calculate the importance of rows. Section 3 discusses the experimental results, and Sect. 4 contains short conclusions.

\section{Main Notions and Tools}

In this section, we consider the main notions, and discuss how to construct the set of upper zeros of the characteristic function for a decision table and how to evaluate the importance of a row.

\subsection{Decision Tables, Tests and Reducts}

A decision table is a rectangular table $T$ whose elements belong to the set $\omega=\{0,1,2, \ldots\}$ of nonnegative integers. Columns of this table are labeled with attributes $f_{1}, \ldots, f_{n}$. Rows of the table are pairwise different, and each row is labeled with a number from $\omega$ (a decision).

A test for $T$ is a subset of attributes (columns) such that at the intersection with these columns any two rows with different decisions are different. A reduct for $T$ is a test for $T$ for which each proper subset is not a test. It is clear that each test has a reduct as a subset.

\subsection{Characteristic Functions}

Let $T$ be a decision table with $n$ columns labeled with attributes $f_{1}, \ldots, f_{n}$. There exists a one-to-one correspondence between $E_{2}^{n}$, where $E_{2}=\{0,1\}$, and the set of subsets of attributes from $T$. Let $\bar{\alpha} \in E_{2}^{n}$ and $i_{1}, \ldots, i_{m}$ be indices of elements from $\bar{\alpha}$ which are equal to 1 . Then the set $\left\{f_{i_{1}}, \ldots, f_{i_{m}}\right\}$ corresponds to the tuple $\bar{\alpha}$.

Let us associate a characteristic function $f_{T}: E_{2}^{n} \rightarrow E_{2}$ with the table $T$. For $\alpha \in E_{2}^{n}$ we have $f_{T}(\bar{\alpha})=1$ if and only if the set of attributes corresponding to $\bar{\alpha}$ is a test for $T$.

We consider now some notions related to monotone Boolean functions. We define a partial order $\leq$ on the set $E_{2}^{n}$ where $n$ is a natural number. Let $\bar{\alpha}=$ $\left(\alpha_{1}, \ldots, \alpha_{n}\right), \bar{\beta}=\left(\beta_{1}, \ldots, \beta_{n}\right) \in E_{2}^{n}$. Then $\bar{\alpha} \leq \bar{\beta}$ if and only if $\alpha_{i} \leq \beta_{i}$ for $i=1, \ldots, n$. The inequality $\bar{\alpha}<\bar{\beta}$ means that $\bar{\alpha} \leq \bar{\beta}$ and $\bar{\alpha} \neq \bar{\beta}$. Two tuples $\bar{\alpha}$ and $\bar{\beta}$ are incomparable if both relations $\bar{\alpha} \leq \bar{\beta}$ and $\bar{\beta} \leq \bar{\alpha}$ do not hold. A function $f: E_{2}^{n} \rightarrow E_{2}$ is called a monotone Boolean function if, for every tuples $\bar{\alpha}, \bar{\beta} \in E_{2}^{n}$, if $\bar{\alpha} \leq \bar{\beta}$ then $f(\bar{\alpha}) \leq f(\bar{\beta})$. 
A tuple $\bar{\alpha} \in E_{2}^{n}$ is called an upper zero of the monotone Boolean function $f$ if $f(\bar{\alpha})=0$ and, for any tuple $\bar{\beta}$ such that $\bar{\alpha}<\bar{\beta}$, we have $f(\bar{\beta})=1$. For any $\bar{\alpha} \in E_{2}^{n}$, the equality $f(\bar{\alpha})=0$ holds if and only if there exists an upper zero $\bar{\beta}$ of $f$ such that $\bar{\alpha} \leq \bar{\beta}$. A tuple $\bar{\alpha} \in E_{2}^{n}$ is called a lower unit of the monotone Boolean function $f$ if $f(\bar{\alpha})=1$ and $f(\bar{\beta})=0$ for any tuple $\bar{\beta}$ such that $\bar{\beta}<\bar{\alpha}$. For any $\bar{\alpha} \in E_{2}^{n}$, the equality $f(\bar{\alpha})=1$ holds if and only if there exists a lower unit $\bar{\beta}$ of $f$ such that $\bar{\beta} \leq \bar{\alpha}$. So the set of upper zeros allows us to describe completely the set of lower units of a monotone Boolean function.

We omit the proof of the following simple statement.

Lemma 1. For any decision table $T$, the characteristic function $f_{T}$ is a monotone Boolean function which does not equal to 0 identically and for which the set of lower units coincides with the set of tuples corresponding to reducts for the table $T$.

\subsection{Canonical Forms of Decision Tables}

Let us associate a decision table $\tau(T)$ with the decision table $T$. The table $\tau(T)$ has $n$ columns labeled with attributes $f_{1}, \ldots, f_{n}$. The first row of $\tau(T)$ is filled by 1. The set of all other rows coincides with the set of all rows of the kind $l\left(\bar{\delta}_{1}, \bar{\delta}_{2}\right)$ where $\bar{\delta}_{1}$ and $\bar{\delta}_{2}$ are arbitrary rows of $T$ labeled with different decisions, and $l\left(\bar{\delta}_{1}, \bar{\delta}_{2}\right)$ is the row containing at the intersection with the column $f_{i}, i=1, \ldots, n$, the number 0 if $\bar{\delta}_{1}$ and $\bar{\delta}_{2}$ have different numbers at the intersection with the column $f_{i}$, and the number 1 otherwise. The first row of $\tau(T)$ is labeled with the decision 1. All other rows are labeled with the decision 2.

We denote by $C(T)$ the decision table obtained from $\tau(T)$ by the removing all rows $\bar{\sigma}$ for each of which there exists a row $\bar{\delta}$ of the table $\tau(T)$ that is different from the first row and satisfies the inequality $\bar{\sigma}<\bar{\delta}$. The table $C(T)$ will be called the canonical form of the table $T$.

Lemma 2. For any decision table $T, f_{T}=f_{C(T)}$.

Proof. One can show that $f_{T}=f_{\tau(T)}$. Let us prove that $f_{\tau(T)}=f_{C(T)}$. It is not difficult to check that $f_{C(T)}(\bar{\alpha})=0$ if and only if there exists a row $\bar{\delta}$ of $C(T)$ labeled with the decision 2 for which $\bar{\alpha} \leq \bar{\delta}$. Similar statement is true for the table $\tau(T)$.

It is clear that each row of $C(T)$ is also a row in $\tau(T)$, and equal rows in these tables are labeled with equal decisions. Therefore if $f_{\tau(T)}(\bar{\alpha})=1$ then $f_{C(T)}(\bar{\alpha})=1$.

Let $f_{C(T)}(\bar{\alpha})=1$. We will show that $f_{\tau(T)}(\alpha)=1$. Let us assume the contrary. Then there exists a row $\bar{\sigma}$ from $\tau(T)$ which is labeled with the decision 2 and for which $\bar{\alpha} \leq \bar{\sigma}$. From the description of $C(T)$ it follows that there exists a row $\bar{\delta}$ from $C(T)$ which is labeled with the decision 2 and for which $\bar{\sigma} \leq \bar{\delta}$. But in this case $\bar{\alpha} \leq \bar{\delta}$ which is impossible. Hence $f_{\tau(T)}(\alpha)=1$ and $f_{\tau(T)}=f_{C(T)}$.

Proposition 1. For any decision table $T$, the set of rows of the table $C(T)$ with the exception of the first row coincides with the set of upper zeros of the function $f_{T}$. 
Proof. Let $\bar{\alpha}$ be an upper zero of the function $f_{T}$. Using Lemma 2 we obtain $f_{C(T)}(\bar{\alpha})=0$. Therefore there exists a row $\bar{\delta}$ in $C(T)$ which is labeled with the decision 2 and for which $\bar{\alpha} \leq \bar{\delta}$. Evidently, $f_{C(T)}(\bar{\delta})=0$. Therefore $f_{T}(\bar{\delta})=0$. Taking into account that $\bar{\alpha}$ is an upper zero of the function $f_{T}$ we conclude that the inequality $\bar{\alpha}<\bar{\delta}$ does not hold. Hence $\bar{\alpha}=\bar{\delta}$ and $\bar{\alpha}$ is a row of $C(T)$ which is labeled with the decision 2 .

Let $\bar{\delta}$ be a row of $C(T)$ different from the first row. Then $f_{C(T)}(\bar{\delta})=0$, and by Lemma $2, f_{T}(\bar{\delta})=0$. Let $\bar{\delta}<\bar{\sigma}$. We will show that $f_{T}(\bar{\sigma})=1$. Let us assume the contrary. Then by Lemma $2, f_{C(T)}(\bar{\sigma})=0$. Therefore there exists a row $\bar{\gamma}$ of $C(T)$ which is labeled with the decision 2 and for which $\bar{\sigma} \leq \bar{\gamma}$ and $\bar{\delta}<\bar{\gamma}$. But this is impossible since any two different rows of $C(T)$ which are labeled with 2 are incomparable. Hence $f_{T}(\bar{\sigma})=1$, and $\bar{\delta}$ is an upper zero of $f_{T}$.

This proposition gives us an efficient way to construct the set $U_{T}$ of upper zeros of the function $f_{T}$. We construct in polynomial time depending on the size of $T$ the canonical form $C(T)$ of $T$. By Proposition 1, the set $U_{T}$ coincides with the set of rows of the table $C(T)$ with the exception of the first row.

\subsection{Importance of Rows}

The idea of evaluation of row importance is the following. We construct the canonical form $C(T)$ of the table $T$ and the set $U_{T}$ of upper zeros of the characteristic function $f_{T}$ corresponding to the table $T$. We remove a row $r$ from the decision table $T$. As a result, we obtain new decision table $T(r)$. We construct the canonical form $C(T(r))$ of the table $T(r)$ and the set $U_{T(r)}$ of upper zeros of the characteristic function $f_{T(r)}$ corresponding to the table $T(r)$. The cardinality $\left|\left(U_{T} \cup U_{T(r)}\right) \backslash\left(U_{T} \cap U_{T(r)}\right)\right|$ of the symmetric difference of the sets $U_{T}$ and $U_{T(r)}$ will be considered as the importance of the row $r$ for the table $T$.

Example 1. Let $T$ be the decision table depicted in Fig. 1 and $r_{4}$ be the fourth row of $T$. We construct tables $C(T), T\left(r_{4}\right)$, and $C\left(T\left(r_{4}\right)\right)$ (see Fig. 1).

$$
\begin{aligned}
& T=\begin{array}{|lll|l|l|}
\hline f_{1} & f_{2} & f_{3} \\
\hline 1 & 1 & 1 & 0 \\
1 & 0 & 0 & 0 \\
0 & 0 & 1 & 1 \\
0 & 0 & 0 & 0 \\
\hline
\end{array} \\
& T\left(r_{4}\right)=\begin{array}{|ccc|c|c|}
f_{1} & f_{2} & f_{3} \\
\hline 1 & 1 & 1 & 0 \\
1 & 0 & 0 & 0 \\
0 & 0 & 1 & 1
\end{array} \quad \quad C\left(T\left(r_{4}\right)\right)=\begin{array}{|llll|}
\hline f_{1} & f_{2} & f_{3} \\
\hline 1 & 1 & 1 & 1 \\
0 & 0 & 1 & 2 \\
0 & 1 & 0 & 2 \\
\hline
\end{array}
\end{aligned}
$$

Fig. 1. Decision table $T$ and tables $C(T), T\left(r_{4}\right)$, and $C\left(T\left(r_{4}\right)\right)$ 
As a result, we have $U_{T}=\{(0,0,1),(1,1,0)\}$ and $U_{T\left(r_{4}\right)}=\{(0,0,1),(0,1,0)\}$. The symmetric difference of the sets $U_{T}$ and $U_{T\left(r_{4}\right)}$ is equal to $\{(0,1,0),(1,1,0)\}$. Therefore the importance of the row $r_{4}$ for the table $T$ is equal to 2 .

Of course, it would be better to compare the sets of reducts of the tables $T$ and $T(r)$ directly. Unfortunately, there are no efficient algorithms for the construction of the set of reducts. So instead of the comparison of the sets of reducts directly, we compare the sets $U_{T}$ and $U_{T(r)}$ which describe completely the sets of reducts for the tables $T$ and $T(r)$, respectively. In particular, the importance of the row $r$ for the table $T$ is equal to 0 if and only if the tables $T$ and $T(r)$ have the same sets of reducts.

\section{Experimental Results}

We did experiments with 10 decision tables from UCI ML Repository [1]. Some preprocessing steps were done before the actual experiments. We removed conditional attributes which have unique value for each row. Each group of identical rows was replaced with a single row labeled with the most common decision for this group. Missing values for an attribute were replaced with a most common value for this attribute. Table 1 contains information about the considered decision tables including the number of rows, the number of conditional attributes, and the existence of important rows - rows for which the importance is greater than zero.

Table 1. Characteristics of decision tables

\begin{tabular}{lrrr}
\hline $\begin{array}{l}\text { Decision } \\
\text { table }\end{array}$ & Rows Attrs Has important \\
rows?
\end{tabular}

For the decision tables BREAST-CANCER, HOUSE-VOTES-84, SPECT-TEST, and ZOO-DATA, we show the results of experiments in the form of range of importance in the left column and the number of rows with importance in this range in the right column.

The decision tables LYMPHOGRAPHY and SOYBEAN-SMALL were not changed during the preprocessing steps. For these tables, for each row with non-zero importance, we show the number of row and its importance. 
For the decision table HOUSE-VOTES-84, we have 15 rows with non-zero importance and 264 rows with zero importance (see Table 2).

Table 2. Importance of rows for HOUSE-VOTES- 84

\begin{tabular}{rr}
\hline $\begin{array}{c}\text { Range of } \\
\text { importance }\end{array}$ & $\begin{array}{c}\text { No. of } \\
\text { rows }\end{array}$ \\
\hline 0 & 264 \\
1 to 5 & 14 \\
14 & 1 \\
\hline
\end{tabular}

For the decision table SPECT-TEST, there are 18 rows with non-zero importance and 151 rows with zero importance (see Table 3).

Table 3. Importance of rows for SPECT-TEST

\begin{tabular}{rr}
$\begin{array}{c}\text { Range of } \\
\text { importance }\end{array}$ & $\begin{array}{c}\text { No. of } \\
\text { rows }\end{array}$ \\
\hline 0 & 151 \\
1 to 5 & 14 \\
6 to 10 & 3 \\
34 & 1 \\
\hline
\end{tabular}

For the decision table ZOO-DATA, there are 44 rows with zero importance and 15 rows with non-zero importance (see Table 4 ).

Table 4. Importance of rows for ZOO-DATA

\begin{tabular}{rr}
\hline $\begin{array}{c}\text { Range of } \\
\text { importance }\end{array}$ & $\begin{array}{c}\text { No. of } \\
\text { rows }\end{array}$ \\
\hline 0 & 44 \\
1 to 5 & 11 \\
9 & 1 \\
12 & 1 \\
13 & 1 \\
20 & 1 \\
\hline
\end{tabular}

For the decision table BREAST-CANCER, there are 262 rows with zero importance and 4 rows with non-zero importance (see Table 5).

For the decision table LYMPHOGRAPHY, there are 41 rows with zero importance and 107 rows with non-zero importance (see Table 6). 
Table 5. Importance of rows for BREAST-CANCER

\begin{tabular}{rr}
$\begin{array}{c}\text { Range of } \\
\text { importance }\end{array}$ & $\begin{array}{c}\text { No. of } \\
\text { rows }\end{array}$ \\
\hline 0 & 262 \\
1 to 5 & 4 \\
\hline
\end{tabular}

Table 6. Importance of rows for LYMPHOGRAPHY

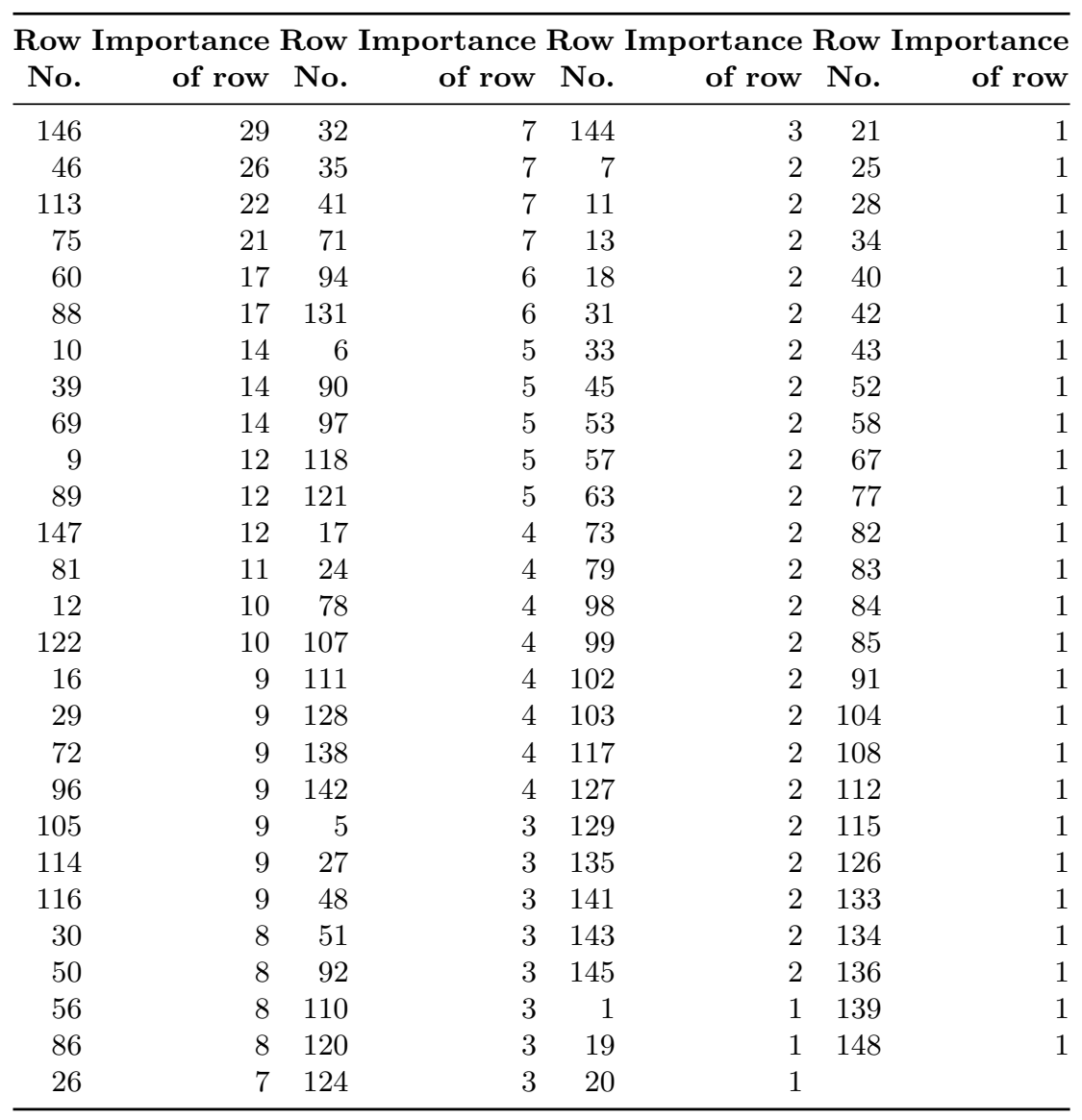

For the decision table SOYBEAN-SMALL, there are 3 rows with zero importance and 44 rows with non-zero importance (see Table 7).

Each of the considered tables has rows that are not important (rows with zero importance). If a row is not important, it does not mean that it does not contain important information: the information contained in the row is "covered" by the information of other rows. If row is important, it means that it contains some unique information relative to other rows, and it deserves special attention, 
Table 7. Importance of rows for SOYBEAN-SMALL

\begin{tabular}{rrrrrrrr}
\hline \multirow{2}{*}{$\begin{array}{r}\text { Row Importance Row Importance Row Importance Row } \\
\text { No. }\end{array}$} & of row & No. & of row & No. & of row & No. & of row \\
\hline 4 & 20 & 42 & 11 & 45 & 7 & 18 & 3 \\
17 & 17 & 1 & 9 & 3 & 6 & 23 & 3 \\
25 & 17 & 7 & 9 & 14 & 5 & 31 & 3 \\
26 & 17 & 9 & 9 & 38 & 5 & 43 & 3 \\
12 & 15 & 24 & 9 & 44 & 5 & 19 & 2 \\
30 & 13 & 34 & 9 & 47 & 5 & 21 & 2 \\
41 & 13 & 5 & 8 & 8 & 4 & 37 & 2 \\
2 & 11 & 27 & 7 & 11 & 4 & 15 & 1 \\
22 & 11 & 29 & 7 & 16 & 4 & 20 & 1 \\
28 & 11 & 32 & 7 & 33 & 4 & 36 & 1 \\
39 & 11 & 35 & 7 & 10 & 3 & 46 & 1 \\
\hline
\end{tabular}

especially if its importance is relatively high (as for the row No. 146 in the decision table LYMPHOGRAPHY which importance is equal to 29).

\section{Conclusions}

In this paper, we proposed a method for the evaluation of importance of rows for decision tables. This allows us to understand how a given row affects the formation of the set of reducts. In the future, we are planning to consider not only the cardinality of the symmetric difference of the sets $U_{T}$ and $U_{T(r)}$ but also other measures of difference of these sets.

\section{Acknowledgements}

Research reported in this publication was supported by King Abdullah University of Science and Technology (KAUST).

\section{References}

1. Lichman, M.: UCI Machine Learning Repository. University of California, Irvine, School of Information and Computer Sciences (2013), http://archive.ics.uci.edu/ml 2. Moshkov, M., Zielosko, B.: Combinatorial Machine Learning - A Rough Set Approach, Studies in Computational Intelligence, vol. 360. Springer, Heidelberg (2011)

3. Pawlak, Z.: Rough Sets - Theoretical Aspects of Reasoning About Data. Kluwer Academic Publishers, Dordrecht (1991)

4. Skowron, A., Rauszer, C.: The discernibility matrices and functions in information systems. In: Słowiński, R. (ed.) Intelligent Decision Support: Handbook of Applications and Advances of the Rough Sets Theory, pp. 331-362. Kluwer Academic Publishers, Dordrecht (1992) 